$11-1-2015$

\title{
The Bayes Factor for Case-Control Studies with Misclassified Data
}

Tzesan Lee

Centers for Disease Control \& Prevention, leetzesan@gmail.com

Follow this and additional works at: http://digitalcommons.wayne.edu/jmasm

Part of the Applied Statistics Commons, Social and Behavioral Sciences Commons, and the Statistical Theory Commons

\section{Recommended Citation}

Lee, Tzesan (2015) "The Bayes Factor for Case-Control Studies with Misclassified Data," Journal of Modern Applied Statistical Methods: Vol. 14 : Iss. 2 , Article 16.

DOI: $10.22237 /$ jmasm/1446351300

Available at: http://digitalcommons.wayne.edu/jmasm/vol14/iss2/16

This Regular Article is brought to you for free and open access by the Open Access Journals at DigitalCommons@WayneState. It has been accepted for inclusion in Journal of Modern Applied Statistical Methods by an authorized editor of DigitalCommons@WayneState. 


\section{The Bayes Factor for Case-Control Studies with Misclassified Data}

\section{Cover Page Footnote}

All the calculations were done on the EXCEL spreadsheet. 


\title{
The Bayes Factor for Case-Control Studies with Misclassified Data
}

\author{
Tzesan Lee \\ Centers for Disease Control \& Prevention \\ Atlanta, GA
}

The question of how to test if collected data for a case-control study are misclassified was investigated. A mixed approach was employed to calculate the Bayes factor to assess the validity of the null hypothesis of no-misclassification. A real-world data set on the association between lung cancer and smoking status was used as an example to illustrate the proposed method.

Keywords: $\quad$ Bayes factor, Misclassification, $p$-value.

\section{Introduction}

Misclassification is a ubiquitous problem in epidemiologic studies. Particularly, it often occurs if the data are obtained from the proxy or surrogate (Nelson, Longstreth, Koesell, and van Belle 1990). Methods for dealing with misclassified data from case-control studies have been widely studied. See, for example, Kleinbaum, Kupper \& Morgenstern (1982), Fleiss, Levin \& Paik (2003), and Rothman, Greenland \& Lash (2008). Almost all studies make an assumption in the beginning that the collected data are misclassified. Yet how to test the validity of this assumption has not been addressed.

These issues can also be considered from a Bayesian perspective. First, the misclassification probabilities are included in both the null and alternative hypothesis. Second, bias-adjusted estimators for the proportion of exposure in cases or controls are presented. Third, the uniform and the Beta distributions are adopted respectively as the prior distribution for the misclassification probability and population proportion parameter in cases or controls. Finally, the lower-bound for the Bayes factor is calculated. A real-world data set was used as an example to illustrate the proposed method. A comparison between the $p$-value and the Bayes factor is made.

Tzesan Lee was retired from the Centers for Disease Control and Prevention and is currently working as President, Applied Math Press, LLC. Email at leetzesan@gmail.com. 
BAYES FACTOR FOR CASE-CONTROL STUDIES MISCLASSIFIED DATA

\section{Methodology}

Consider the data for case-control studies given in Table 1. The random variable $E^{*}$ denotes the classified surrogate for the true exposure variable $E$, while the variable $D$ indicates the disease status of the subjects with $D=1$ and $D=0$ representing cases and controls respectively. Suppose that $E^{*}$ is misclassified, but $D$ is not misclassified.

Table 1. Case-control studies with misclassified data

\begin{tabular}{rcr}
\hline $\begin{array}{r}\text { Classified exposure } \\
\text { status }\end{array}$ & $\begin{array}{r}\text { Group of subjects } \\
\text { (cases) }\end{array}$ & $\boldsymbol{D = 0}$ (controls) \\
\hline$E^{*}=1$ (exposed) & $\mathrm{n}_{11}$ & $\mathrm{n}_{10}$ \\
$E^{*}=0$ (unexposed) & $\mathrm{n}_{01}$ & $\mathrm{n}_{00}$ \\
Sample size & $\mathrm{n}_{[1]}$ & $\mathrm{n}_{[0]}$ \\
\hline
\end{tabular}

It is well known that the traditional sample proportion estimator of the exposed group given by

$$
\hat{p}_{i}=n_{j i} / n_{[i]}, \hat{q}_{i}=1-\hat{p}_{i}
$$

In terms of the sensitivity and specificity defined by

$$
\begin{gathered}
\varphi_{i}=\operatorname{Pr}\left(E^{*}=1 \mid E=1, D=i\right), \bar{\varphi}_{i}=1-\varphi_{i} \\
\psi_{i}=\operatorname{Pr}\left(E^{*}=1 \mid E=0, D=i\right), \bar{\psi}_{i}=1-\psi_{i}
\end{gathered}
$$

it was shown (Lee, 2009) that

$$
\begin{gathered}
E\left(\hat{p}_{i}\right)=\varphi_{i} p_{i}+\left(1-\psi_{i}\right) q_{i}=p_{i} \cdot \Delta_{i}+1-\psi_{i} \\
E\left(\hat{q}_{i}\right)=\left(1-\varphi_{i}\right) p_{i}+\psi_{i} q_{i}=q_{i} \cdot \Delta_{i}+1-\varphi_{i}
\end{gathered}
$$




\section{TZESAN LEE}

From Equations 4 and 5 it is seen that the traditional sample proportion

estimators, $\hat{p}_{i}$ and $\hat{q}_{i}$, are no longer unbiased. By solving Equations 4 and 5 with the left-side $E\left(\hat{p}_{i}\right)$ or $E\left(\hat{q}_{i}\right)$ being replaced by $\hat{p}_{i}$ or $\hat{q}_{i}$, it follows

$$
\begin{aligned}
& \breve{p}_{i}=\left(\psi_{i}-\hat{q}_{i}\right) / \Delta_{i}, \\
& \breve{q}_{i}=\left(\varphi_{i}-\hat{p}_{i}\right) / \Delta_{i},
\end{aligned}
$$

where

$$
\Delta_{i}=\varphi_{i}+\psi_{i}-1, i=0,1
$$

Equations 6 and 7 are called the bias-adjusted proportion (BAP) estimators of $p_{i}$ and $q_{i}$. The BAP estimators are said to be admissible if they are greater than zero but less than one plus their sum equals to one. Evidently, the following constraints are required to be imposed on the sensitivity and specificity in order for Equations 6 and 7 to be admissible (Lee, 2009):

$$
\begin{aligned}
\varphi_{i} & >\hat{p}_{i}, \\
\psi_{i} & >\hat{q}_{i}, \\
\varphi_{i}+\psi_{i} & >1 .
\end{aligned}
$$

A concern is aimed at testing whether the given data in Table 1 are misclassified - whether the exposure rates for cases and control are the same. This can be tested through the hypothesis testing which is formulated as follows:

$$
H_{0}: \varepsilon_{R D}=0 \text { versus } H_{1}: \varepsilon_{R D} \neq 0 \text {, }
$$

where $\varepsilon_{R D}=p_{1}-p_{0}$, the subscript " $R D$ " means the rate difference. However, Equation 10 can't be used to test whether the observed data of Table 1 are misclassified. In order to test if the data are misclassified, the hypotheses of Equation 10 has to be enlarged by including the misclassification probabilities associated with both cases and controls given as follows:

$$
H_{0}: \varepsilon_{R D}=0, \bar{\varphi}_{i}=\bar{\psi}_{i}=0 \text { versus } H_{1}: \varepsilon_{R D} \neq 0, \bar{\varphi}_{i} \neq 0, \bar{\psi}_{i} \neq 0, i=0,1 \text {, }
$$




\section{BAYES FACTOR FOR CASE-CONTROL STUDIES MISCLASSIFIED DATA}

To test the hypotheses of Equation 11, a mixed Bayesian approach is taken to tackle this problem (Kass \& Raftery, 1995).

Let

$$
\breve{\varepsilon}_{R D}=\breve{p}_{1}-\breve{p}_{0}-\varepsilon_{R D}
$$

It can be shown

$$
\begin{gathered}
E\left(\breve{\varepsilon}_{R D}\right)=0, \\
\operatorname{Var}\left(\breve{\varepsilon}_{R D}\right)=\operatorname{Var}\left(\breve{p}_{1}\right)+\operatorname{Var}\left(\breve{p}_{0}+\varepsilon_{R D}\right) \\
=\sum_{i=0}^{1}\left(p_{i} \cdot \Delta_{i}+1-\psi_{i}\right)\left(q_{i} \cdot \Delta_{i}+1-\varphi_{i}\right) \cdot n_{[i]}^{-1}
\end{gathered}
$$

Define

$$
\breve{x}_{R D}=\breve{\varepsilon}_{R D}^{2} / \operatorname{Var}\left(\breve{\varepsilon}_{R D}\right)
$$

To assess the evidence in favor of supporting the null against the alternative hypothesis of Equation 11, the Bayes factor for favoring $H_{0}$ relative $H_{1}$ from using Equation 15 can be calculated as follows:

$$
B^{g}\left(\breve{x}_{R D}\right)=\frac{f\left(\breve{x}_{R D} \mid H_{0}\right)}{m_{g}\left(\breve{x}_{R D}\right)}
$$

where

$$
m_{g}\left(\breve{x}_{R D}\right)=\iint_{R \times \Omega} f\left(\breve{x}_{R D} \mid H_{1}\right) \prod_{i=0}^{1} h_{0}\left(\varphi_{i}, \psi_{i}\right) g\left(p_{i}, q_{i}\right) d \varphi_{i} d \psi_{i} d p_{i} d q_{i}
$$

$f\left(\breve{x}_{R D} \mid H_{1}\right)$ is the central chi-square distribution with one degree of freedom, $g\left(p_{i}, q_{i}\right)=\Gamma(\eta+\tau) p_{i}^{\eta-1} q_{i}^{\tau-1} /[\Gamma(\eta) \Gamma(\tau)]$, the beta distribution with the parameters $\eta$ and $\tau$ over $[0,1]$, and $h_{0}\left(\varphi_{i}, \psi_{i}\right)=\left[\bar{\varphi}_{i} \bar{\psi}_{i}\right]^{-1}$ is the uniform distribution 


\section{TZESAN LEE}

over $\Omega_{i}=\left[a_{i}, 1\right] \times\left[b_{i}, 1\right]$, where $a_{i}$ and $b_{i}$ are specified in the Appendix. Although the posterior marginal probability density function of $m_{g}$ (Equation 17 ) depends on two hyper-parameters $\eta$ and $\tau$, a Bayes/non-Bayes compromise rather than a type III hyper-distribution for $\eta$ and $\tau$ is adopted to estimate $\eta$ and $\tau$ (Good \& Crook, 1974). As a result, the parameters $\eta$ and $\tau$ are estimated by employing the likelihood method. The maximum likelihood estimators for $\eta$ and $\tau$ and the relative maximum value of $\mathrm{m}_{\mathrm{g}}$ of Equation 17 are denoted respectively by $\left(\eta_{\max }, \tau_{\max }\right)$ and $m_{g}^{\max }=m_{g}\left(\eta_{\max }, \tau_{\max }\right)$. Thus, define the lower bound of the Bayes factor (Equation 16) as follows:

$$
\underline{B}^{g}=f\left(\breve{x}_{R D} \mid H_{0}\right) / m_{g}^{\max }
$$

The details of calculating Equation 18 are given in the Appendix.

\section{Example}

Although there is some evidence of a greater than average risk in some occupations to have the lung cancer, these occupations could not account for the general increase in pulmonary cancer. It is thought of interest to select a particular population group, homogeneous economically, with little occupational exposure to respiratory irritants and with equal access to diagnostic facilities. Physicians are believed to represent such a group. Wynder and Cornfield (1953) reported a study on the exposure to tobacco and other possible respiratory irritants of 63 physicians with lung cancer and 133 physicians with cancers in areas where respiratory irritants are not believed to play a part. Among these 133 physicians, 43 cases were cancer of stomach and kidney, 45 cases cancer of colon and lymphoma, and 45 cases cancer of bladder, leukemia and sarcoma. The data in Table 2 is taken from Cornfield (1956) who only used 43 cases from cancer of stomach and kidney as a control group. The non-smoker is defined to be those who smoked the equivalent of less than 1 cigarette a day. Here it is of interest to test whether the data concerning the smoking status in Table 2 for both cases and controls are misclassified. 
BAYES FACTOR FOR CASE-CONTROL STUDIES MISCLASSIFIED DATA

Table 2. The data of physicians with and without lung cancer by smoking status

\begin{tabular}{rrr}
\hline Smoking status & Lung cancer patients & Controls \\
\hline Smoker & 60 & 32 \\
Nonsmoker & 3 & 11 \\
Total & 63 & 43 \\
\hline
\end{tabular}

Before calculating the Bayes factor, the data in Table 2 are first to be checked if the two required conditions are satisfied before using the formula derived in the Appendix. Because $\hat{p}_{1}=0.952381>\hat{p}_{0}=0.744186$ and $\hat{\sigma}_{\hat{p}_{1}}=\sqrt{n_{[1]}^{-1} \hat{p}_{1} \hat{q}_{1}}=0.027>\hat{\sigma}_{\hat{p}_{0}}=\sqrt{n_{[0]}^{-1} \hat{p}_{0} \hat{q}_{0}}=0.067$, where $n_{[1]}=63, n_{[0]}=43$, the two required conditions are indeed being satisfied; hence it was free to use the formula in the Appendix. Let $a_{i}=\hat{p}_{i}+0.005$ and $b_{i}=\hat{q}_{i}+0.005, i=0,1$, be substituted into Equations A17 to A11, it follows that $\dot{M}_{[1,1,0,0]}=1.1011, M_{[1,0,1,0]}=0.0828, M_{[1,0,0,1]}=-0.0037, \dot{M}_{[1,1,0,1]}=0.0513$, $M_{[1,0,1,1]}=1.2369, \dot{M}_{[0,1,0,0]}=1.1169, M_{[0,0,1,0]}=0.6287, M_{[0,0,0,1]}=-0.0567$, $\dot{M}_{[0,1,0,1]}=0.4819$, and $M_{[0,0,1,1]}=4.8652$. Then, substituting the above information into Equations $\mathrm{A} 12$ and $\mathrm{A} 14$, this leads to that $N_{0}=0.1957, N_{1}=5.4652$, $\mathrm{N}_{2}=-31.4597, R_{0}=0.0016, R_{1}=0.1967, R_{2}=-0.0041, R_{3}=0.0704, R_{4}=0.234$, $R_{5}=-0.0252, R_{6}=-0.1988$, and $a=133.5876$. Again, by substituting the above information into Equations A13 and A16, it follows that

$$
m_{g}^{(1)}(\eta, \tau) \equiv \frac{-400.8(\eta+\tau)\left\{\begin{array}{l}
\eta \tau(\eta+\tau)[0.003 \eta(\eta+\tau)-0.002] \\
+0.017 \eta \tau(\eta+\tau)+0.002 \eta]+0.009 \tau
\end{array}\right\}+5.97 \tau}{2 \sqrt{\eta \tau} \eta^{2}(\eta+\tau)^{3}}
$$

and

$$
m_{g}^{(2)}(\eta, \tau) \equiv \frac{2.33 \eta \tau(\eta+\tau)^{2}+2.23 \tau(\eta+\tau)-3.82}{2[\sqrt{\eta \tau}(\eta+\tau)]^{3}}
$$

Consequently, $m_{g}(\eta, \tau)$ was readily obtained from substituting Equations 19 and 20 into Equation A17. 


\section{TZESAN LEE}

To find the relative maximum of $m_{g}(\eta, \tau)$, the 2-dimensional unit square $[0,1] \times[0,1]$ was partitioned into 100 lattice points $(0.1,0.1),(0.1,0.2), \ldots,(1.0,0.9),(1.0,1.0)$ and then evaluated the function value of $m_{g}(\eta, \tau)$ at these lattice points. After identifying the proximity of the relative maximum a finer neighborhood was then searched to locate it. Equation A17 was found to have a unique relative maxima: $m_{g}^{\max }(0.14,1.0)=2.15$. The value of $f\left(\breve{x}_{R D} \mid H_{0}\right)$ was evaluated directly from the probability density function of the central chi-square distribution with one degree of freedom; hence we have $f\left(\breve{x}_{R D} \mid H_{0}\right)=6.4 \times 10^{-6}$. After dividing the value of $f\left(\breve{x}_{R D} \mid H_{0}\right)=6.4 \times 10^{-6}$ by $m_{g}^{\max }=2.15$, we thus obtained the lower bound of the Bayes factor given by $\underline{B}^{g}\left(\breve{x}_{R D}\right)=3.0 \times 10^{-6}$.

Since $\quad \breve{x}_{R D} \mid H_{0}=\hat{x}_{R D}=\hat{p}_{D}^{2} / \operatorname{Var}\left(\hat{p}_{D}\right)=19.1 \quad\left(p\right.$-value $\left.=1.2 \times 10^{-5}\right), \quad$ where $\hat{p}_{D} \equiv \hat{p}_{1}-\hat{p}_{0}$, the null hypothesis $H_{0}$ was rejected for Table 2 . Yet, the evidence from the lower bound of the Bayes factor $\left(\underline{B}^{g}\left(\breve{x}_{R D}\right)=3.0 \times 10^{-6}\right)$ was in favor of supporting $H_{1}$ (Equation 11) by at most a factor of " $3.3 \times 10^{5}$ to 1 ". Hence the data in Table 2 are likely to be misclassified.

\section{Discussion}

Although both the $p$-value and the Bayes factor rejected the null hypothesis $\mathrm{H}_{0}$ with respect to the data in Table 2, the $p$-value seemed much inclined to reject the null hypothesis $H_{0}$ in Equation 10 rather than that in Equation 11. In other words, the $p$-value is inadequate to reject the null hypothesis in Equation 11. This study provides another example to corroborate the $p$-value fallacy (Goodman 1999a, Goodman 1999b).

Because the Beta distribution which is the conjugate family of the binomial distribution was used as the prior distributions, the Bayes factor could of course change accordingly if other family of distributions is used as the prior distribution (Delampady \& Berger, 1990).

The derivation of the formula provided in the Appendix was based on the

two assumptions: (i) $p_{1}>p_{0}$, and (ii) $\sigma_{\hat{p}_{1}}=\sqrt{n_{[1]}^{-1} p_{1} q_{1}}<\sigma_{\hat{p}_{0}}=\sqrt{n_{[0]}^{-1} p_{0} q_{0}}$. These two assumptions can be verified if it is valid by substituting the crude prevalence estimator $\left(\hat{p}_{i}, i=0,1\right)$ into the inequality. Should the both of the two assumptions fail to be satisfied, all we need to do is to switch the index accordingly for cases 


\section{BAYES FACTOR FOR CASE-CONTROL STUDIES MISCLASSIFIED DATA}

and controls before using the formula provided in the Appendix. However, if only one of the assumptions is violated, Equation A4 has to be revised accordingly.

\section{References}

Askey, R. A. \& Roy, R. (2010). Gamma function, NIST handbook of mathematical functions (pp.135). F. W. Olver, D. W. Lozier, R. F. Boisvert, C. W. Clark (Eds.), United Kingdom: National Institute of Standards and Technology, Cambridge University Press.

Cornfield, J. (1956). A statistical problem arising from retrospective studies. In Proceedings of the third Berkeley symposium on mathematical statistics and probability (Vol. 4, pp. 135-148). Berkeley: University of California Press.

Delampady, M. \& Berger, J. O. (1990). Lower bounds on Bayes factors for multinomial distributions with application to chi-squared tests of fit. The Annals of Statistics, 18(3), 1295-1316. doi:10.1214/aos/1176347750

Fleiss, J., Levin, B. \& Paik, M. C. (2003). Statistical methods for rates and proportions ( ${ }^{\text {rd }}$ ed.). New York: John Wiley \& Sons.

Good, I. J. \& Crook, J. F. (1974). The Bayes/non-Bayes compromise and the multinomial distribution. Journal of American Statistical Association, 69(347), 711-720. doi:10.2307/2286006

Goodman, S. N. (1999a). Toward evidence-based medical statistics. 1: The P-value fallacy, Annals of Internal Medicine, 130(12), 995-1004. doi:10.7326/0003-4819-130-12-199906150-00008

Goodman, S. N. (1999b). Toward evidence-based medical statistics. 2: The Bayes factor, Annals of Internal Medicine, 130(12), 1005-1013. doi:10.7326/0003-4819-130-12-199906150-00019

Kass, R. E. and Raftery, A. E. (1995). Bayes factor, Journal of American Statistical Association, 90(430), 773-795. doi:10.1080/01621459.1995.10476572

Kleinbaum, D. G., Kupper, L. L. \& Morgenstern, H. (1982). Epidemiologic research: Principles and quantitative methods. Belmont, CA: Lifetime Learning.

Lee, T-S. (2009). Bias-adjusted exposure odds ratio for misclassified data, The Internet Journal of Epidemiology, 6(2), 1-19.

Mietinen, O. \& Nurminen, M. (1985). Comparative analysis of two rates. Statistics in Medicine, 4(2), 213-226. doi:10.1002/sim.4780040211 


\section{TZESAN LEE}

Nelson, L. M., Longstreth, W. T., Koesell, T. D., \& van Belle, G. (1990). Proxy respondents in epidemiologic research. Epidemiologic Reviews, 12(1), 71-86.

Rothman, K. J., Greenland, S. \& Lash, T. L. (2008). Modern epidemiology ( $3^{\text {rd }}$ ed.). Philadelphia, PA: Lippincott Williams \& Wilkins.

Wynder, E. L. \& Cornfield, J. (1953). Cancer of the lung in physicians, New England Journal of Medicine, 248(11), 441-444.

doi:10.1056/NEJM195303122481101

\section{Appendix}

By applying the quadratic approximation to the probability density function of the central chi-square distribution with one degree of freedom in Equation 17, we have

$$
\begin{aligned}
f\left(\breve{x}_{R D} \mid \varepsilon_{R D}, \varphi_{0}, \psi_{0}, \varphi_{1}, \psi_{1}\right) & =\frac{1}{\sqrt{2 \pi}} \breve{x}_{R D}^{-\frac{1}{2}} e^{-\frac{1}{2} \breve{x}_{R D}} \\
& \approx \frac{1}{\sqrt{2 \pi}} \cdot \frac{1}{\sqrt{\breve{x}_{R D}}}\left(1-\frac{1}{2} \breve{x}_{R D}+\frac{1}{8} \breve{x}_{R D}^{2}\right) \\
& =\frac{1}{\sqrt{2 \pi}}\left[\frac{\sqrt{\operatorname{Var}\left(\breve{\varepsilon}_{R D}\right)}}{\breve{\varepsilon}_{R D}}-\frac{1}{2} \frac{\breve{\varepsilon}_{R D}}{\sqrt{\operatorname{Var}\left(\breve{\varepsilon}_{R D}\right)}}+\frac{1}{8}\left(\frac{\breve{\varepsilon}_{R D}}{\sqrt{\operatorname{Var}\left(\breve{\varepsilon}_{R D}\right)}}\right)^{3}\right],
\end{aligned}
$$

(A1)

where $\breve{\varepsilon}_{R D}$ and $\operatorname{Var}\left(\breve{\varepsilon}_{R D}\right)$ are given by Equations 12 and 14, respectively.

By using the linear approximation:

$$
\left[\left(1-\varepsilon_{R D}^{-1}\left(\breve{p}_{1}-\breve{p}_{0}\right)\right]^{-1} \approx 1+\varepsilon_{R d}^{-1}\left(\breve{p}_{1}-\breve{p}_{0}\right),\right.
$$

it follows that 
BAYES FACTOR FOR CASE-CONTROL STUDIES MISCLASSIFIED DATA

$$
\begin{aligned}
\frac{\sqrt{\operatorname{Var}\left(\breve{\varepsilon}_{R d}\right)}}{\breve{\varepsilon}_{R d}} & =\frac{\sqrt{\begin{array}{l}
\Delta_{1}^{-2} n_{[1]}^{-1}\left(p_{1} \Delta_{1}+1-\psi_{0}^{-2}\right)\left(q_{1} \Delta_{10]}+1-\varphi_{1}\right) \\
\left.p_{0} \Delta_{0}+1-\psi_{0}\right)\left(q_{0} \Delta_{0}+1-\varphi_{0}\right)
\end{array}}}{\breve{p}_{1}-\breve{p}_{0}-\varepsilon_{R D}} \\
& =-\frac{\sqrt{A}}{\varepsilon_{R D}} \cdot \frac{\sqrt{1+A^{-1}\left\{\sum_{i=0}^{1} n_{[i]}^{-1} \Delta_{i}^{-1}\left[\left(1-p_{i} \varphi_{i}-q_{i} \psi_{i}\right)+\Delta_{i}^{-1} \bar{\varphi}_{i} \bar{\psi}_{i}\right]\right\}}}{1-\varepsilon_{R D}^{-1}\left(\breve{p}_{1}-\breve{p}_{0}\right)} \\
& =-I^{-1} \cdot \varepsilon_{R D}^{-1} \cdot \sqrt{1+I^{2} J} \cdot\left[1-\varepsilon_{R D}^{-1}\left(\breve{p}_{1}-\breve{p}_{0}\right)\right]^{-1} \\
& \approx-I^{-1} \cdot \varepsilon_{R D}^{-1}\left(1+\frac{1}{2} I^{2} J\right)\left[1+\varepsilon_{R D}^{-1}\left(\breve{p}_{1}-\breve{p}_{0}\right)\right] \\
& =-I^{-1} \cdot \varepsilon_{R D}^{-1}\left[1+\varepsilon_{R D}^{-1}\left(\breve{p}_{1}-\breve{p}_{0}\right)+\frac{1}{2} I^{2} J+\frac{1}{2} \varepsilon_{R D}^{-1} I^{2} J\left(\breve{p}_{1}-\breve{p}_{0}\right)\right] \\
& =-I^{-1} \cdot \varepsilon_{R D}^{-1}\left\{\begin{array}{l}
1+\varepsilon_{R D}^{-1}\left[\Delta_{1}^{-1} u\left(\varphi_{1}\right)-\Delta_{0}^{-1} u\left(\varphi_{0}\right)\right]+\frac{1}{2} I^{2} J \\
+\frac{1}{2} \varepsilon_{R D}^{-1} I^{2} J\left[\Delta_{1}^{-1} u\left(\varphi_{1}\right)-\Delta_{0}^{-1} u\left(\varphi_{0}\right)\right]
\end{array}\right\}
\end{aligned}
$$

where

$$
\begin{aligned}
A & =n_{[1]}^{-1} p_{1} q_{1}+n_{[0]}^{-1} p_{0} q_{0} \\
I & =A^{-\frac{1}{2}} \\
J & =\sum_{i=0}^{1} K_{i} \\
K_{i} & =n_{[1]}^{-1}\left[\begin{array}{l}
\Delta_{1}^{-1}\left(1-p_{i} \varphi_{i}-q_{i} \psi_{i}\right) \\
\left.+\Delta_{i}^{-2} \bar{\varphi}_{i} \bar{\psi}_{i}\right)
\end{array}\right]=n_{[1]}^{-1}\left[\begin{array}{l}
-q_{i}+\Delta_{i}^{-1} s\left(\varphi_{i}\right) \\
+\Delta_{i}^{-2} t\left(\varphi_{i}\right)
\end{array}\right] \\
s\left(\varphi_{i}\right) & =q_{i}\left(2 \varphi_{i}-1\right) \\
t\left(\varphi_{i}\right) & =\varphi_{i}\left(1-\varphi_{i}\right) \\
u\left(\varphi_{i}\right) & =\hat{p}_{i}-\varphi_{i}
\end{aligned}
$$

By using the quadratic approximation on $\varepsilon_{R D}^{-1}, I^{-1}$ and $I$, we have by assuming that $p_{1}>p_{0}$ and $n_{[1]}^{-1} p_{1} q_{1}<n_{[0]}^{-1} p_{0} q_{0}$ 


\section{TZESAN LEE}

$$
\begin{aligned}
& \varepsilon_{R D}^{-1} \approx p_{1}^{-1}+p_{0} p_{1}^{-2}+p_{0}^{2} p_{1}^{-3} \\
& I^{-1} \approx \frac{1}{\sqrt{n_{[0]}}}\left[\sqrt{p_{0} q_{0}}+\frac{1}{2} \frac{n_{[0]}}{n_{[1]}} \cdot \frac{p_{1} q_{1}}{\sqrt{p_{0} q_{0}}}-\frac{1}{8} \frac{n_{[0]}^{2}}{n_{[1]}^{2}} \cdot \frac{\left(p_{1} q_{1}\right)^{2}}{\left(p_{0} q_{0}\right)^{\frac{3}{2}}}\right] \\
& I \equiv \frac{1}{\sqrt{A}} \approx \sqrt{\frac{n_{[0]}}{p_{0} q_{0}}}\left[1-\frac{1}{2} \cdot \frac{n_{[0]} p_{1} q_{1}}{n_{[1]} p_{0} q_{0}}+\frac{3}{8}\left(\frac{n_{[0]} p_{1} q_{1}}{n_{[1]} p_{0} q_{0}}\right)^{2}\right]
\end{aligned}
$$

For fixed $i=0,1$ let

$$
M_{[i, j, k, l]} \equiv \int_{a_{i} b_{i}}^{1}\left[s^{j}\left(\varphi_{i}\right) t^{k}\left(\varphi_{i}\right) u^{l}\left(\varphi_{i}\right)\right] / \Delta_{i}^{j+2 k+l} d \psi_{i} d \varphi_{i}
$$

where $a_{i}=\hat{p}_{i}+0.005, b_{i}=\hat{q}_{i}+0.005, s\left(\varphi_{i}\right), t\left(\varphi_{i}\right)$ and $u\left(\varphi_{i}\right)$ are all defined in Equation A3. Let us calculate some of Equation A5 which will be needed later. For $j=1, k=l=0$ we have

$$
\begin{aligned}
M_{[i, 1,0,0]} & \equiv \int_{a_{i}}^{1} \int_{b_{i}}^{1}\left[s\left(\varphi_{i}\right) / \Delta_{i}\right] d \psi_{i} d \varphi_{i}=\int_{a_{i}}^{1} s\left(\varphi_{i}\right)\left[\ln \varphi_{i}-\ln \left(\varphi_{i}-\bar{b}_{i}\right)\right] d \varphi_{i} \\
& =\int_{a_{i}}^{1}\left\{\left[\left(s^{\prime}(0) \varphi_{i}+s(0)\right] \ln \varphi_{i}-\left[\begin{array}{l}
\left.\left.s^{\prime}\left(\bar{b}_{i}\right)\left(\varphi_{i}-\bar{b}_{i}\right)\right] \ln \left(\varphi_{i}-\bar{b}_{i}\right)\right\} d \varphi_{i} \\
+s\left(\bar{b}_{i}\right)
\end{array}\right]\right.\right. \\
& =q_{i} \dot{M}_{[i, 1,0,0]}
\end{aligned}
$$

where $\delta_{i}=a_{i}+b_{i}-1, \bar{b}_{i}=1-b_{i}$, and

$$
\begin{aligned}
& \dot{M}_{[i, 1,0,0]} \equiv \delta_{i}^{2} \ln \delta_{i}-a_{i}^{2} \ln a_{i}-b_{i}^{2} \ln b_{i}+a_{i} \ln a_{i} \\
&+\left(2 \bar{b}_{i}-1\right)\left(\delta_{i} \ln \delta_{i}-b_{i} \ln b_{i}+b_{i}-\delta_{i}\right)+\frac{1}{2}\left(1+a_{i}^{2}+b_{i}^{2}-\delta_{i}^{2}\right)
\end{aligned}
$$

For $j=l=0, k=1$ we have 
BAYES FACTOR FOR CASE-CONTROL STUDIES MISCLASSIFIED DATA

$$
\begin{aligned}
& M_{[i, 0,1,0]}=\int_{a_{i} b_{i}}^{1} \int_{b_{i}}^{1}\left[t\left(\varphi_{i}\right) / \Delta_{i}^{2} d \psi_{i} d \varphi_{i}\right. \\
& =\int_{a_{i}}^{1}\left[\begin{array}{l}
\frac{\frac{1}{2} t^{\prime \prime}\left(\bar{b}_{i}\right)\left(\varphi_{i}-\bar{b}_{i}\right)^{2}+t^{\prime}\left(\bar{b}_{i}\right)\left(\varphi_{i}-\bar{b}_{i}\right)+t\left(\bar{b}_{i}\right)}{\varphi_{i}-\bar{b}_{i}} \\
-\frac{\frac{1}{2} t^{\prime \prime}(0) \varphi_{i}^{2}+t^{\prime}(0) \varphi_{i}+t(0)}{\varphi_{i}}
\end{array}\right] d \varphi_{i} \\
& =\sum_{m=1}^{3}\left(d_{m[i, 0,1,0]}+e_{m[i, 0,1,0]}\right)=b_{i} \bar{b}_{i} \ln \left(b_{i} / \delta_{i}\right)
\end{aligned}
$$

where $\bar{a}_{i}=1-a_{i}$, and

$$
\begin{aligned}
& d_{1[i, 0,1,0]}=-\frac{1}{2} \bar{a}_{i}\left(b_{i}+\delta_{i}\right), d_{2[i, 0,1,0]}=\bar{a}_{i}\left(1-2 \bar{b}_{i}\right), d_{3[i, 0,1,0]}=b_{i} \bar{b}_{i} \ln \left(b_{i} / \delta_{i}\right), \\
& e_{1[i, 0,1,0]}=\frac{1}{2} \bar{a}_{i}\left(1+a_{i}\right), e_{2[i, 0,1,0]}=-\bar{a}_{i}, e_{3[i, 0,1,0]}=0 .
\end{aligned}
$$

For $j=k=0, l=1$ we have

$$
\begin{aligned}
M_{[i, 0,0,1]} & \equiv \int_{a_{i}}^{1} \int_{b_{i}}^{1} \frac{u\left(\varphi_{i}\right)}{\Delta_{i}} d \psi_{i} d \varphi_{i} \\
& =-\hat{p}_{i} a_{i} \ln a_{i}+\left(\hat{q}_{i}-b_{i}\right)\left(b_{i} \ln b_{i}-\delta_{i} \ln \delta_{i}\right) \\
& +\frac{1}{2}\left(a_{i}^{2} \ln a_{i}+b_{i}^{2} \ln b_{i}-\delta_{i}^{2} \ln \delta_{i}\right) \\
& -\frac{1}{4}\left(a_{i}^{2}+b_{i}^{2}-\delta_{i}^{2}-1\right)-\bar{a}_{i} \bar{b}_{i}
\end{aligned}
$$

For $j=l=1, k=0$ we have 


\section{TZESAN LEE}

$$
\begin{aligned}
& M_{[i, 1,0,1]}=\int_{a_{i}}^{1} \int_{b_{i}}^{1} \frac{s\left(\varphi_{i}\right)}{\Delta_{i}} \cdot \frac{u\left(\varphi_{i}\right)}{\Delta_{i}} d \psi_{i} d \varphi_{i} \\
& -\int_{a_{i}}^{1}\left[\begin{array}{c}
\frac{\frac{1}{2} v_{1}^{\prime \prime}\left(\bar{b}_{i}\right)\left(\varphi_{i}-\bar{b}_{i}\right)^{2}+v_{i}^{\prime}\left(\bar{b}_{i}\right)\left(\varphi_{i}-\bar{b}_{i}\right)+v\left(\bar{b}_{i}\right)}{\varphi_{i}-\bar{b}_{i}} \\
-\frac{\frac{1}{2} v^{\prime \prime}(0) \varphi_{i}^{2}+v^{\prime}(0) \varphi_{i}+v(0)}{\varphi_{i}}
\end{array}\right] d \varphi_{i} \\
& =\sum_{m=1}^{3}\left(d_{m[i, 1,0,1]}+e_{m[i, 1,0,1]}\right)=q_{i} \dot{M}_{[i, 1,0,1]}
\end{aligned}
$$

where

$$
\begin{aligned}
v_{i}\left(\varphi_{i}\right) & =s\left(\varphi_{i}\right) u\left(\varphi_{i}\right)=q_{i}\left(2 \varphi_{i}-1\right)\left(\hat{p}_{i}-\varphi_{i}\right), \\
d_{1[i, 1,0,1]} & =-q_{i} \bar{a}_{i}\left(b_{i}+\delta_{i}\right), d_{2[i, 1,0,1]}=q_{i} \bar{a}_{i}\left[1+2\left(\hat{p}_{i}-\bar{b}_{i}\right)\right], \\
d_{3[i, 1,0,1]} & =q_{i} \bar{b}_{i}\left[1+2\left(\hat{p}_{i}-\bar{b}_{i}\right)-\hat{p}_{i}\right] \ln \left(b_{i} / \delta_{i}\right), \\
e_{1[i, 1,0,1]} & =q_{i} \bar{a}_{i}\left(1+a_{i}\right), e_{2[i, 1,0,1]}=-q_{i}\left(1+2 \hat{p}_{i}\right) \bar{a}_{i}, e_{3[i, 1,0,1]}=\hat{p}_{i} q_{i} \ln a_{i}, \\
\dot{M}_{[i, 1,0,1]} & \equiv \bar{b}_{i}\left[\left(1+\hat{p}_{i}-2 \bar{b}_{i}\right) \ln \left(b_{i} / \delta_{i}\right)+\hat{p}_{i} \ln a_{i}\right]
\end{aligned}
$$

For $j=0, k=l=1$ we have 


$$
\begin{aligned}
& M_{[i, 0,1,1]}=\int_{a_{i} b_{i}}^{1} \frac{t\left(\varphi_{i}\right)}{\Delta_{i}^{2}} \cdot \frac{u\left(\varphi_{i}\right)}{\Delta_{i}} d \psi_{i} d \varphi_{i} \\
& =\frac{1}{2} \int_{a_{i}}^{1}\left[\begin{array}{l}
\frac{\frac{1}{6} v_{2}^{\prime \prime \prime}\left(\bar{b}_{i}\right)\left(\varphi_{i}-\bar{b}_{i}\right)^{3}+\frac{1}{2} v_{2}^{\prime \prime}\left(\bar{b}_{i}\right)\left(\varphi_{i}-\bar{b}_{i}\right)^{2}}{+v_{2}^{\prime}\left(\overline{b_{i}}\right)\left(\varphi_{i}-\overline{b_{i}}\right)+v_{2}(\bar{b})_{i}} \\
\left(\varphi_{i}+\bar{b}_{i}\right)^{2}
\end{array}\right] d \varphi_{i} \\
& =\sum_{m=1}^{4}\left(d_{m[i, 0,1,1]}+e_{m[i, 0,1,1]}\right) \\
& =2 \bar{a}_{i} \bar{b}_{i}+\frac{1}{2}\left\{\begin{array}{l}
{\left[3 \bar{b}_{i}^{2}-2\left(1+\hat{p}_{i}\right) \bar{b}_{i}+\hat{p}_{i}\right] \ln \left(b_{i} / \delta_{i}\right)} \\
+b_{i} \bar{a}_{i} \bar{b}_{i}\left(\hat{p}_{i}-\bar{b}_{i}\right)\left(b_{i}+\delta_{i}\right) /\left(b_{i} \delta_{i}\right)^{2}
\end{array}\right\}
\end{aligned}
$$

where

$$
\begin{aligned}
& v_{2}\left(\varphi_{i}\right)=t\left(\varphi_{i}\right) u\left(\varphi_{i}\right)=\varphi\left(1-\varphi_{i}\right)\left(\hat{p}_{i}-\varphi_{i}\right), \\
& d_{1[i, 0,1,1]}=\frac{1}{4} \bar{a}_{i}\left(b_{i}+\delta_{i}\right), d_{1[i, 0,1,1]}=\frac{1}{2} \bar{a}_{i}\left[3 \bar{b}_{i}-\left(1+\hat{p}_{i}\right)\right], \\
& d_{3[i, 0,1,1]}=\frac{1}{2}\left[3 \bar{b}_{i}^{2}-2\left(1+\hat{p}_{i}\right) \bar{b}_{i}+\hat{p}_{i}\right] \ln \left(b_{i} / \delta_{i}\right), \\
& d_{4[i, 0,1,1]}=\frac{1}{2} b_{i} \bar{b}_{i}\left(\hat{p}_{i}-\bar{b}_{i}\right) \bar{a}_{i}\left(b_{i}+\delta_{i}\right) /\left(b_{i} \delta_{i}\right)^{2}, \\
& e_{1[i, 0,1,1]}=-\frac{1}{4} \bar{a}_{i}\left(1+a_{i}\right), e_{2[i, 0,1,1]}=\frac{1}{2}\left(1+\hat{p}_{i}\right) \bar{a}_{i}, e_{3[i, 0,1,1]}=\frac{1}{2} \hat{p}_{i} \ln a_{i}, e_{4[i, 0,1,1]}=0 .
\end{aligned}
$$

Note that in all of the above calculations I first integrate with respect to $\psi_{i}$ and then integrate with respect to $\varphi_{i}$ by employing the Taylor's series expansion to expand the function about $\varphi_{i}=\bar{b}_{i}$ or 0 .

Now we are ready to calculate the marginal probability density function of Equation A1 one by one 


\section{TZESAN LEE}

$$
\begin{aligned}
& \left(I^{-1} \varepsilon_{R D}^{-1} \varsigma^{-1}+I^{-1} \varepsilon_{R D}^{-2}\left(\begin{array}{l}
\bar{a}_{0} \bar{b}_{0} M_{[1,0,0,1]} \\
-\bar{a}_{1} \bar{b}_{1} M_{[0,0,0,1]}
\end{array}\right)\right.
\end{aligned}
$$

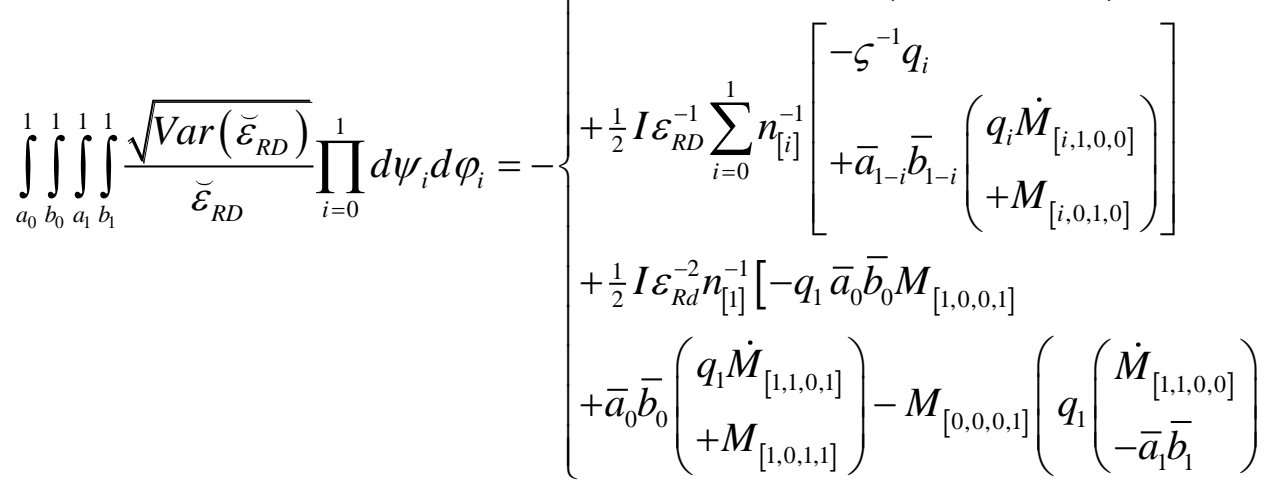

$$
\begin{aligned}
& =-\left\{\begin{array}{c}
I^{-1} \varepsilon_{R D}^{-1} \varsigma^{-1}+I^{-1} \varepsilon_{R D}^{-2} R_{0} \\
+\frac{1}{2} I \varepsilon_{R d}^{-1}\left[\begin{array}{c}
n_{[1]}^{-1}\left(q_{1} R_{1}+\bar{a}_{0} \bar{b}_{0} M_{[1,0,1,0]}\right. \\
+n_{[0]}^{-1}\left(q_{0} R_{2}+\bar{a}_{1} \bar{b}_{1} M_{[0,0,1,0]}\right.
\end{array}\right] \\
+\frac{1}{2} I \varepsilon_{R D}^{-2}\left[n_{[1]}^{-1}\left(q_{1} R_{3}+R_{4}\right)\right.
\end{array}\right.
\end{aligned}
$$

where

$$
\begin{aligned}
R_{0} & =\bar{a}_{0} \bar{b}_{0} M_{[1,0,0,1]}-\bar{a}_{1} \bar{b}_{1} M_{[0,0,0,1]}, R_{1} \equiv \bar{a}_{0} \bar{b}_{0} \dot{M}_{[1,1,0,0]}-\varsigma^{-1}, R_{2} \equiv \bar{a}_{1} \bar{b}_{1} M_{[1,0,1,0]}-\varsigma^{-1} \\
R_{3} & \equiv M_{[0,0,0,1]}\left(\bar{a}_{1} \bar{b}_{1}-\dot{M}_{[1,1,0,0]}\right)+\bar{a}_{0} \bar{b}_{0}\left(\dot{M}_{[1,1,0,1]}-M_{[1,0,0,1]}\right) \\
R_{4} & \equiv \bar{a}_{0} \bar{b}_{0} M_{[1,0,1,1]}-M_{[0,0,0,1]} M_{[1,0,1,0]}, \\
R_{5} & \equiv M_{[1,0,0,1]}\left(\dot{M}_{[[0,1,0,0]]}-\bar{a}_{0} \bar{b}_{0}\right)+\bar{a}_{1} \bar{b}_{1}\left(M_{[0,0,0,1]}-\dot{M}_{[0,1,0,1]}\right) \\
R_{6} & \equiv M_{[1,0,0,1]} M_{[0,0,1,0]}-\bar{a}_{1} \bar{b}_{1} \cdot M_{[0,0,1,1]} \\
\Rightarrow &
\end{aligned}
$$




$$
\begin{aligned}
& m_{g}^{(1)} \equiv \omega \varsigma \iint_{R \times \Omega} \frac{\sqrt{\operatorname{Var}\left(\breve{\varepsilon}_{R d}\right)}}{\breve{\varepsilon}_{R d}} \prod_{i=0}^{1} p_{i}^{\eta-1} q_{i}^{\tau-1} d p_{i} d q_{i} d \psi_{i} d \varphi_{i} \\
& =-\varsigma\left\{\begin{array}{l}
\frac{3 N_{0} \varsigma^{-1} \tau}{\sqrt{\eta \tau}}+\left(9 N_{0} R_{0} \tau+\frac{3}{2} N_{1}\left(n_{[1]}^{-1} R_{1} \tau+\bar{a}_{0} \bar{b}_{0} M_{[1,0,1,0]}\right) \frac{1}{\eta(\eta+\tau) \sqrt{\eta \tau}}\right. \\
+\frac{3}{2} N_{1}\left(n_{[0]}^{-1} R_{2} \tau+\bar{a}_{1} \bar{b}_{1} M_{[1,0,1,0]}\right) \frac{1}{\eta(\eta+\tau)^{2} \sqrt{\eta \tau}} \\
+\frac{9}{2} N_{1} \tau\left[\eta^{2}(\eta+\tau)^{3} \sqrt{\eta \tau}\right]^{-1}\left[n_{[1]}^{-1}\left(R_{3}(\eta+\tau)+R_{4}\right)+n_{[0]}^{-1}\left(R_{5}(\eta+\tau)+R_{6}\right)\right]
\end{array}\right\} \\
& =\frac{-3 \varsigma(\eta+\tau)\left\{\begin{array} { l } 
{ 2 N _ { 0 } \eta \tau ( \eta + \tau ) \cdot } \\
{ ( \varsigma ^ { - 1 } \eta ( \eta + \tau ) + 3 R _ { 0 } ) }
\end{array} N _ { 1 } \left[\begin{array}{l}
\eta \tau(\eta+\tau)\left(n_{[1]}^{-1} R_{1}+n_{[0]}^{-1} R_{2}\right) \\
+\eta\left(n_{[1]}^{-1} \bar{a}_{0} \bar{b}_{0}+n_{[0]}^{-1} \bar{a}_{1} \bar{b}_{1}\right) M_{[1,0,1,0]}+3 .
\end{array}\right.\right.}{2 \sqrt{\eta \tau} \eta^{2}(\eta+\tau)^{3}} \\
& \left.\left.\tau\left(n_{[1]}^{-1} R_{3}+n_{[0]}^{-1} R_{5}\right)\right]\right\}-9 N_{1} \varsigma \tau\left(n_{[1]}^{-1} R_{4}+n_{[0]}^{-1} R_{6}\right)
\end{aligned}
$$

where for $i, j, k, l=0,1 M_{[i, j, k, \ell]}$ and $\dot{M}_{[i, j, k, \ell]}$ are given respectively by Equations A6-A10,

$$
\begin{aligned}
& \omega=\{\Gamma(\eta+\tau) /[\Gamma(\eta) \Gamma(\tau)]\}^{2}, \\
& \varsigma=\left(\bar{a}_{0} \bar{b}_{0} \bar{a}_{1} \bar{b}_{1}\right)^{-1}, \bar{a}_{i}=1-a_{i}, \bar{b}_{i}=1-b_{i}, \\
& N_{0}=n_{[0]}^{-\frac{1}{2}}\left(1+\frac{1}{2} n_{[1]}^{-1} n_{[0]}-\frac{1}{8} n_{[1]}^{-2} n_{[0]}^{2}\right), \\
& N_{1}=n_{[0]}^{\frac{1}{2}}\left(1-\frac{1}{2} n_{[0]} n_{[1]}^{-1}+\frac{3}{8} n_{[0]}^{2} n_{[1]}^{-2}\right)
\end{aligned}
$$

On the other hand, by integrating the following equation with respect to $\varphi_{i}, \psi_{i}, i=0,1$ 


\section{TZESAN LEE}

$$
\begin{aligned}
\frac{\breve{\varepsilon}_{R D}}{\sqrt{\operatorname{Var}\left(\breve{\varepsilon}_{R D}\right)}} & =\frac{\breve{p}_{1}-\breve{p}_{0}-\varepsilon_{R D}}{\sqrt{A}}\left(1-\frac{J}{2 A}\right) \\
& =I\left(\frac{u\left(\varphi_{1}\right)}{\Delta_{1}}-\frac{u\left(\varphi_{0}\right)}{\Delta_{0}}-\varepsilon_{R D}\right)-\frac{1}{2} I^{3}\left(\frac{u\left(\varphi_{1}\right)}{\Delta_{1}}-\frac{u\left(\varphi_{0}\right)}{\Delta_{0}}-\varepsilon_{R D}\right) J
\end{aligned}
$$

This leads to

$$
\begin{aligned}
& \int_{a_{0}}^{1} \int_{b_{0}}^{1} \int_{a_{1}}^{1} \int_{b_{1}}^{1} \frac{\breve{\varepsilon}_{R D}}{\sqrt{\operatorname{Var}\left(\breve{\varepsilon}_{R D}\right)}} \prod_{i=0}^{1} d \psi_{i} d \varphi_{i}=I\left(R_{0}-\varsigma^{-1} \varepsilon_{R D}\right) \\
& -\frac{1}{2} I^{3}\left\{\begin{array}{c}
n_{[1]}^{-1}\left[\begin{array}{l}
q_{1} R_{3}+R_{4} \\
-\varepsilon_{R D}\left(q_{1} R_{1}+\bar{a}_{0} \bar{b}_{0} M_{[1,0,1,0]}\right)
\end{array}\right] \\
+n_{[0]}^{-1}\left[\begin{array}{l}
q_{0} R_{5}+R_{6} \\
-\varepsilon_{R D} \cdot\left(q_{0} R_{2}+\bar{a}_{1} \bar{b}_{1} M_{[0,0,1,0]}\right)
\end{array}\right]
\end{array}\right\}
\end{aligned}
$$

Further, we obtain by integrating Equation A15 with respect to $p_{i}, q_{i}, i=0,1$

$$
\begin{aligned}
m_{g}^{(2)} & \equiv \omega \varsigma \iint_{\Omega \times R} \frac{\breve{\varepsilon}_{R D}}{\sqrt{\operatorname{Var}\left(\breve{\varepsilon}_{R D}\right)}} \prod_{i=0}^{1} p_{i}^{\eta-1} q_{i}^{\tau-1} d p_{i} d q_{i} d \psi_{i} d \varphi_{i} \\
& =\varsigma\left\{\frac{N_{1} R_{0}}{(\eta+\tau) \sqrt{\eta \tau}}-\frac{1}{2}\left[\frac{N_{2}\left(n_{[1]}^{-1} R_{3}+n_{[0]}^{-1} R_{5}\right)}{\eta(\eta+\tau)^{2} \sqrt{\eta \tau}}+\frac{N_{2}\left(n_{[1]}^{-1} R_{4}+n_{[0]}^{-1} R_{6}\right)}{\eta \tau(\eta+\tau)^{3} \sqrt{\eta \tau}}\right]\right. \\
& \left.\left.=\frac{\varsigma\left\{2 N_{1} R_{0} \eta \tau(\eta+\tau)^{2}-N_{2}\left[\tau(\eta+\tau)\left(n_{[1]}^{-1} R_{3}+n_{[0]}^{-1} R_{5}\right)\right]\right\}}{2\left[\sqrt{\eta \tau}(\eta+\tau) n_{[1]}^{-1} R_{4}+n_{[0]}^{-1} R_{6}\right.}\right]\right\}
\end{aligned}
$$

where $\varsigma, N_{1}, R_{0}$, and $R_{j}, j=3,4,5,6$ are given respectively by Equations $\mathrm{A} 12$ and A14, and

$$
N_{2} \equiv \sqrt{n_{[0]}^{3}}\left(1-\frac{3 n_{[0]}}{2 n_{[1]}}-\frac{3 n_{[0]}^{2}}{8 n_{[1]}^{2}}-\frac{n_{[0]}^{3}}{8 n_{[1]}^{3}}+\frac{45 n_{[0]}^{4}}{64 n_{[1]}^{4}}-\frac{27 n_{[0]}^{5}}{128 n_{[1]}^{5}}+\frac{27 n_{[0]}^{6}}{512 n_{[1]}^{6}}\right)
$$




\section{BAYES FACTOR FOR CASE-CONTROL STUDIES MISCLASSIFIED DATA}

Note that in calculating Equations A13 and A16 I used an approximation on the Gamma function: $\Gamma(z+a) / \Gamma(z+b) \approx z^{a-b}$ (Askey \& Roy, 2010).

By integrating Equation 12 with respect to $\left(\varphi_{i}, \psi_{i}\right)$ first and then $\left(p_{i}, q_{i}\right)$ for $i=0,1$ we obtain $m_{g}(\eta, \tau)$ by substituting Equations A13 and A16 into Equation A17:

$$
m_{g}(\eta, \tau)=(2 \pi)^{-\frac{1}{2}}\left\{m_{g}^{(1)}(\eta, \tau)-\frac{1}{2} m_{g}^{(2)}(\eta, \tau)+\frac{1}{8}\left[m_{g}^{(2)}(\eta, \tau)\right]^{3}\right\}
$$

